\title{
Modeling Filtering Penalties in ROADM-based Networks with Machine Learning for QoT Estimation
}

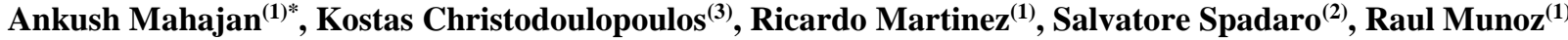 \\ ${ }^{(1)}$ Centre Tecnològic de Telecomunicacions de Catalunya (CTTC/CERCA), ${ }^{(2)}$ Polytechnic University of Catalonia (UPC), Barcelona, Spain, \\ ${ }^{(3)}$ Nokia Bell Labs, Stuttgart, Germany \\ *ankush.mahajan@cttc.cat
}

\begin{abstract}
Monitoring 3dB bandwidth and other spectrum related parameters at ROADMs provides information about quality of their filters. We propose a machine-learning model to estimate end-toend filtering penalty for more accurate QoT estimation of future connections. () 2020 The Author(s)

OCIS codes: 060.4256 Networks, network optimization; 060.4510 Optical communications
\end{abstract}

\section{Introduction}

Reconfigurable Optical Add/Drop Multiplexers (ROADMs) are the key switching elements of deployed core and metro optical networks [1]. Several implementations of ROADMs are possible using optical devices including MUX/DEMUX, optical splitters/combiners, wavelength blockers and wavelength selective switches (WSSs) [2]. The use of WSS provides the advantages of colorless, and/or directionless and/or contentionless node operation and higher degree design, making them industry's choice for current generation ROADMs [1, 2].

Generally, a signal that traverses a ROADM node suffers from filtering penalty due to the involved WSS(s) resulting in signal quality of transmission (QoT) degradation. Over longer paths the cascade of ROADM filters introduces stronger filtering, which narrows the signal's transmission bandwidth (BW) [3]. During the lightpath provisioning , such optical filtering penalty is covered inside margins of the QoT estimation tool (or Qtool). Research works $[3,4]$ confirmed that ROADM node penalty (in OSNR) increases exponentially with the number of nodes and depends on the modulation format and the grid spacing. An analytical model based on a higher order SNR-OSNR relation to capture cascaded filtering effects is presented in [5]. These prior works focus on characterization of the cascade (either by looping single filter, or by replacing cascaded filters with a tunable BW filter) and do not focus on the identification of the quality of the individual filters. Although these works consent that filtering penalty is nonlinear to the number of filters, still there are uncertainties in these penalties. The misalignment of the filters to the grid, deviations in filters shape and $3 \mathrm{~dB}$ BW are quite common in deployed networks and are covered in the aforementioned Qtool margins. Such issues are expected to exacerbate in disaggregated optical networks where ROADM/filters and Tx. lasers could come from multiple vendors with diverse characteristics [5, 6]. In disaggregated (at node level) networks margins of 3.5-5dB (core) and 3-3.5dB (metro) would be required [6] mainly due to uncertainties/variability of the multi-vendor components; the filtering penalty of ROADM nodes play a significant part in those increased margins. In a network (either single or multi-vendor), connections traverse different ROADMs and experience different degrees of filtering penalty. Using cheap optical channel monitors (OCM) [7] we can understand the filters alignment to the grid and their shape. Such understanding could be used to correct the Tx/cascade alignment and improve the QoT of existing connections [8] or improve QoT estimation and reduce margin for future connection establishments.

In light of the above herein, we firstly examine filters behavior under uncertainties and corresponding penalties. We then propose a Machine Learning (ML) regression model based on link formulation that leverages monitoring data of established connections to accurately estimate end-to-end filtering penalty for new connection requests.

\section{Methodology and Proposed Solution}

ROADMs consists of amplifiers and filters to boost and route the signal. Now, consider a channel having central wavelength $\lambda$ that is routed through multiple ROADMs over a path $p$ before finally detected at the receiver. This can be viewed as a line transmission system with filters along the path ( 1 or 2 per ROADM for Broadcast and Select-B\&S, or Switch/Route and Select-S\&S architecture). We denote the spectrum of an individual filter in the path as $\boldsymbol{F}_{i}(\lambda), i \in$ $\boldsymbol{p}$ and by $p_{i}$ the part of the path before $i^{\text {th }}$ filter. We also denote by $\boldsymbol{C}_{\boldsymbol{p}_{i}}(\lambda)$ the overall filter spectrum before $i^{\text {th }}$ filter due to the cascade of previous filters over $p_{i}$ and by $\boldsymbol{C}_{p}(\lambda)$ the overall filter spectrum at end of the path (Rx.), is given by:

$$
C_{\boldsymbol{p}_{\boldsymbol{i}}}=\prod_{\boldsymbol{k} \in \boldsymbol{p}_{\boldsymbol{i}}} F_{k}(\lambda), \quad C_{p}(\lambda)=\prod_{\boldsymbol{k} \in \boldsymbol{p}} F_{k}(\lambda)
$$

Assuming that $i^{\prime}$ is the next filter after $i$ on the path, by monitoring $C_{p_{i}}(\lambda)$ and $C_{p_{i \prime}}(\lambda)$ we can calculate $F_{i}(\lambda)$ which gives us valuable information about key properties (filter shape and alignment to the grid) of the $i^{\text {th }}$ filter at wavelength $\lambda$. Typically, such properties hold for all $\lambda \mathrm{s}$ of the same filter. From monitored $C_{p_{i}}(\lambda)$, we extract a set of features $j$ that reflects the properties of cascade before $\mathrm{i}$, denoted by $C_{i}^{j}(\lambda)$, such as $3 \mathrm{~dB} \mathrm{BW}$, cascaded filter central frequency, signal distribution parameters $\left(1^{\mathrm{st}} / 2^{\text {nd }}\right.$ order statistical moments), or $C_{i}^{j}(\lambda)=\left\{C_{i}^{3 d B}(\lambda), C_{i}^{f c}(\lambda), \ldots, C_{i}^{\text {sym. }}(\lambda)\right\}$. Figure 1(a) shows 

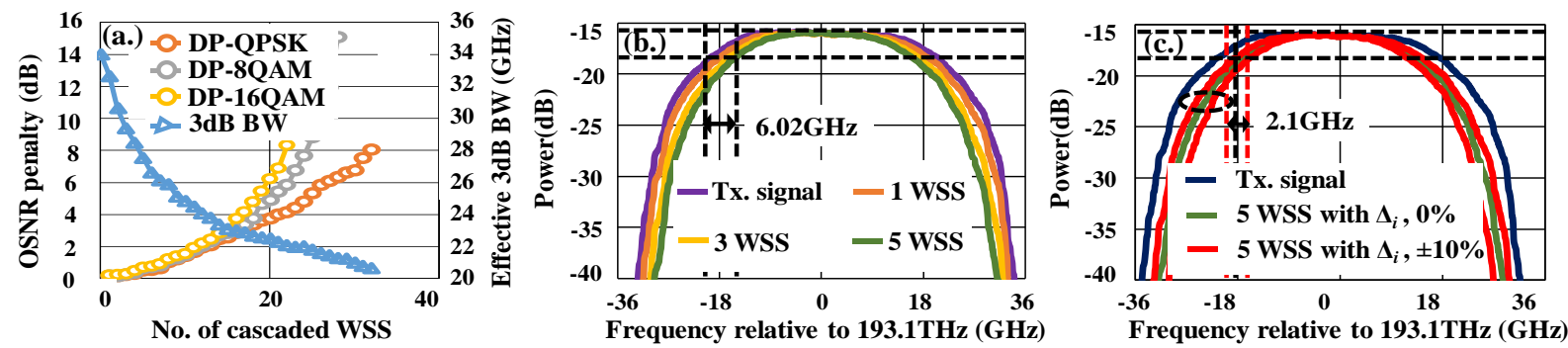

Fig. 1: (a) OSNR penalty \& $3 \mathrm{~dB} \mathrm{BW}$, (b) spectrum for increasing number of cascaded WSSs (identical $F_{i}(\lambda)$ for all WSSs), (c) spectrum for $3 \mathrm{~dB}$ BW uncertainty $\Delta_{i}^{3 d B}= \pm 10 \%$ (non-identical $F_{i}(\lambda)$ ), resulting in $\sim 2.1 \mathrm{GHz}$ of uncertainty at $5^{\text {th }}$ cascaded WSS, compare to Fig. 1(b) (red region) the non-linear/exponential degradation of one such feature, i.e. $3 \mathrm{~dB} \mathrm{BW}, C_{i}^{3 \mathrm{~dB}}(\lambda)$, in a cascade of identical filters $(3 \mathrm{~dB}$ $\mathrm{BW}=37.5 \mathrm{GHz}, 2^{\text {nd }}$ order Gaussian shape) obtained with simulations in VPI. It also shows the OSNR (in dB) penalty for three modulation formats (@ 32Gbaud, $\alpha=0.1$ ). Focusing on spectral response, Fig. 1(b) shows the spectral shape of the signal $C_{p_{i}}(\lambda)$, which degrades as the number of cascade increases, even for identical filters. We observed a $3 \mathrm{~dB}$ BW degradation of $C_{i}^{3 d B}(\lambda)=6.02 \mathrm{GHz}$ after 5 identical filters. However, in real networks slight variations are typical within the spectral responses even of identical filters, while such variations would exacerbate in disaggregated scenarios if filters/ROADMs come from different vendors. Such variations result in uncertainties in the features $C_{i}^{j}(\lambda)$, which we denote as $\Delta_{i}^{j}(\lambda)=\left\{\Delta_{i}^{3 d B}(\lambda), \Delta_{i}^{f c}(\lambda), \ldots, \Delta_{i}^{s y m}(\lambda)\right\}$. Fig. $1(\mathrm{c})$, shows the resulted $C_{p}(\lambda)$ for a $3 \mathrm{~dB}$ BW uncertainty of $\Delta_{i}^{3 d B}= \pm 10 \%$ per filter after a cascade of 5 filters, simulated in VPI. We observed $\sim 2.1 \mathrm{GHz}$ uncertainty in the $3 \mathrm{~dB}$ $\mathrm{BW}, C_{p}^{3 d B}(\lambda)$, at the end of the cascade/path, which contributes to inaccurate filter penalty estimation.
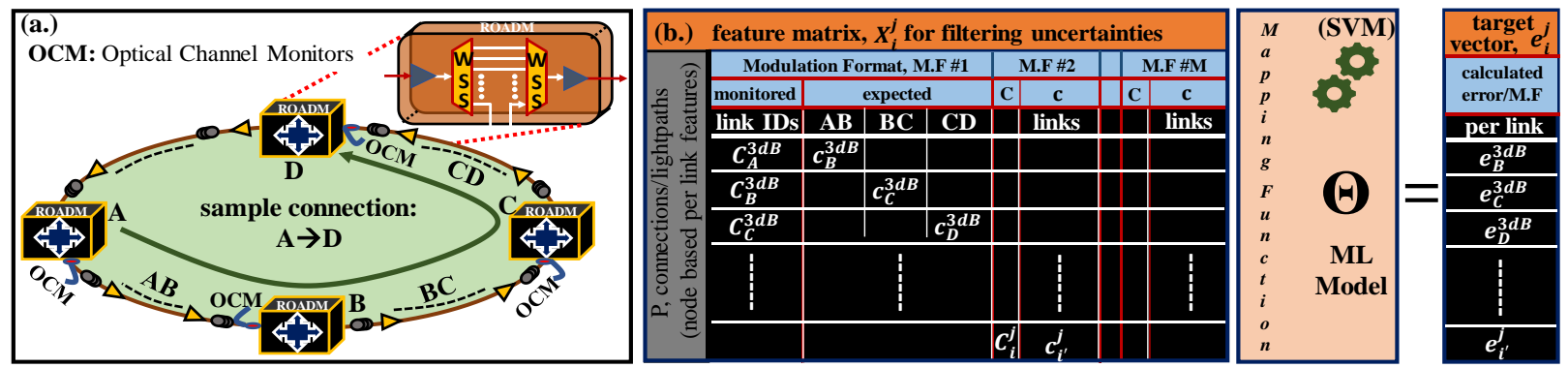

Fig. 2: (a) distributed OCM locations for a sample network (4 nodes, with established connection from A to D) with switch \& select, S\&S ROADM architecture (in inset), (b) end to end (link formulation approach based) ML model along with feature matrix, $X$ and target vector, $e$

Focusing on filter penalty modeling, a standard Qtool (denoted by $Q_{s}$ ) calculates the features (e.g. $3 \mathrm{~dB}$ BW) and filter penalty along a path assuming identical filters (Fig. 1(a)) and uses a high margin on top to account for penalties from inaccuracies (filter alignment and shape). By exploiting monitoring information, we can extend $Q_{s}$ to understand the actual network state and behavior of deployed filters, reduce inaccuracies and lower the margin for new connections $[9,10]$. Today cheap OCMs [7] can be installed at ROADM nodes to monitor $C_{p_{i}}(\lambda)$ on any channel. Each ROADM includes WSS/filters depending on its degree and architecture. Depending on add/drop or crossing direction, different filters are encountered in the network, hence in the following we account the filters $i$ on a per link bases. We assume an optical network with established connections and their attributes denoted by $P$. We use OCMs to monitor $C_{i}\left(\lambda^{P}\right)$, and extract $C_{i}^{j}\left(\lambda^{P}\right)$ before each ROADM node along the paths of established connections $(P)$. So, the features $C_{i}^{j}\left(\lambda^{P}\right.$ suppress to simplify the notation) serve as the ground truth and are stored in Qtool database. Typically, a standard Qtool would start from the Tx. parameters and iteratively calculate the cascaded features for a specific filter $F_{i}$ with no uncertainty, $\Delta_{i}^{j}=0$, down the path until the receiver and add a margin for not accounting for uncertainties. So the standard Qtool, $Q_{s}$, includes a function that takes the features before ROADM $i, c_{i}^{j}$, and calculates the expected features after ROADM $i, Q_{s}\left(c_{i}^{j}, F_{i}\right)$ assuming no uncertainty $\Delta_{i}^{j}=0$ (Fig. 1(a) shows such a $Q_{s}$ function). Since we have OCM information $C_{i}^{j}$, we can correct $Q_{s}$ and reduce the margin as follows. We denote the expected feature as $c_{i}^{j}=Q_{s}\left(C_{i}^{j}, F_{i}\right)$ and the monitored-expected error as $e_{i \prime}^{j}=C_{i}^{j}-c_{i \prime}^{j}$, which is due to unknown uncertainties $\Delta_{i}^{j}$. Then we extract a per link features matrix, $X_{i}^{j}=f\left(C_{i}^{j}, c_{i}^{j}\right)$. Our goal is to identify a per link dependent error function, $\Theta\left(X_{i}^{j}\right) \approx e_{i}^{j}$, which maps the features matrix $X_{i}^{j}$ to the error $e_{i}^{j}$. We rely on ML for training and fitting of $X$ on $e$ and finding $\Theta$. Fig. 2(b) shows the features matrix $X$ utilizing OCM data $C_{i}^{j}, Q_{s}$ expected features $c_{i}^{j}$ and error $e$ for the toy network of Fig.2(a). Assuming a new connection request $p \notin P$ using wavelength $l$, we start with its transmission spectrum parameters $c_{p_{0}}^{j}(l)$, use $Q_{s}$ to obtain the expected feature set $c_{p_{1}}^{j}(l)$ after the ingress node, extract $X_{p_{0}}^{j}(l)$ correct that by calculating 
$e_{p_{0}}^{j}=\Theta\left(X_{p_{0}}^{j}(l)\right)$, and repeat that link by link down the path until destination. The estimation error will be identified once we establish the connection, monitor $C_{p_{i}}^{j}(l)$ at available OCMs and compare it to estimations by the above algorithm.

\section{Results \& Discussion}

To quantify the benefits of developed QoT estimator with more accuracy and reduced margins, we considered DT topology with 12 nodes and 40 bidirectional links with lengths from 48 to $458 \mathrm{~km}$ as (inset Fig. 3(a)). The span was assumed to be standard single-mode fiber and span length equal to $80 \mathrm{~km}$. Each demand is carried by one wavelength and modulated at 32Gbaud with \{QPSK, 8-QAM, 16-QAM $\}$ modulation formats leading to $\{100,150,200\}$ Gbps of datarate. The frequency slot size was assumed to be $12.5 \mathrm{GHz}$ (fixed) and we allocated 3 spectrum slots of $12.5 \mathrm{GHz}$.
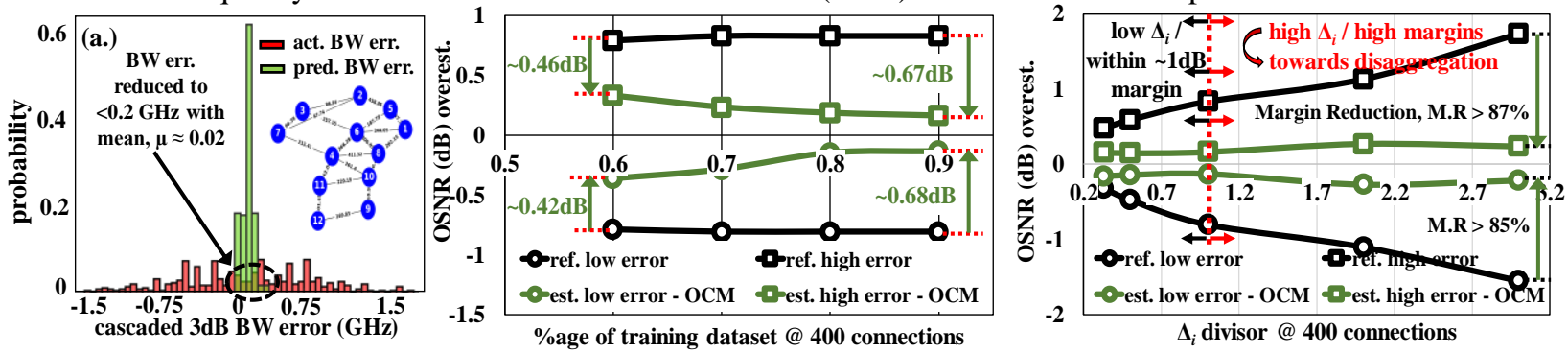

Fig. 3: (a) end to end effective $3 \mathrm{~dB}$ BW error and reduced ML estimated error, (b) errors ( \pm ve) in OSNR (dB): reference (black), ML max overestimation with OCM data (green), (c) new margin \& their reduction with different $\Delta_{i}$ intensities

We assumed a network with OCMs installed at each node (Fig. 2(a)) and generated monitoring data (ground truth), by randomly applying small $\Delta_{i}$ (resulted in end to end $\pm 1.5 \mathrm{GHz} 3 \mathrm{~dB}$ variation) reflecting Tx. \& filters-grid mismatch, and small variations in filters shape. The corresponding OSNR penalties are also distributed in $\pm v e$ sides depending upon $\Delta_{i} .+$-ve penalties result in upper/lower bound for design margins and we call them as, "high/low margin or errors". We assumed a stable network state, where a set of connections is established and the aim is to provision a new set of new connections. To do so, we divided the connections into two sets for training and testing, assumed to be the established and the new connections, respectively. Then from the training dataset, we calculated errors, $e$ based on the expected and monitored $3 \mathrm{~dB}$ BW, central wavelength, symmetry, link IDs, route etc. information. We also generated, the per link feature matrix, $X$. We used support vector machine, SVM, fitting technique with gaussian kernel function, to train our ML model, and we achieved least max. MSE of $\sim 0.02 \mathrm{~dB}$ on predicted OSNR at a maximum load of 400 connections (200 times average @ 400 connections). Fig. 3(a) shows the calculated error in 3dB BW at a load of 400 connections and also the error reduction in BW (from $\pm 1.5 \mathrm{GHz} \rightarrow \sim 0.18 \mathrm{GHz}$ ) with trained SVM. Fig. 3(b) reflects these accurate (per link) estimation of 3dB BW in end-to-end accurate estimation of filtering/OSNR penalty (green lines). Fig. 3(b) shows that at $90 \% / 10 \%$ train/test split, maximum error reduction/accuracy improvement was $\sim 0.67 \mathrm{~dB}$ for high error and $\sim 0.68 \mathrm{~dB}$ for low errors, respectively. These are the new reduced high and low margins for the actual and unknown filtering uncertainties. For high/low margin, we found an overall reduction of $80.4 / 83.4 \%$ at a load of 400 connections. We also varied $\Delta_{i}$ (multiplied by a factor of $1 / 3$ to 3 ) and estimated high and low margins/errors at a fixed load of 400 connections. In Fig. 3(c), the high $\Delta_{i}$ scenario (> 1dB, right of red dashed line) reflects ROADMs nodes with higher uncertainty, which are expected in disaggregated/multi-vendor networks. As expected, higher reference margins are required there, and our accurate modeling results in more pronounced savings that reach $>85 \%$ and $>1.5 \mathrm{~dB}$ on both high and low margins.

\section{Conclusion}

We proposed ML model to estimate end-to-end penalty generated at ROADM nodes due to filter spectral uncertainties $\&$ their cascaded effects. Harnessing monitored data and leveraging ML techniques, we estimated QoT accurately for new connections with max. of $\sim 0.68 \mathrm{~dB}$ of OSNR accuracy and $>80 \%$ reduction in related margin.

Acknowledgements: Authors would like to thank Karsten Schuh and Camille Delezoide of Nokia Bell Labs for technical discussions on filter modelling. This work is a part of H2020-MSCA, ONFIRE project supported by EU, grant agreement No. 765275.

\section{References}

[1] M. Filer, et al., "N-degree ROADM Architecture Comparison: B\&S vs. R\&S in 120 Gb/s DP-QPSK Transmission Systems," OFC, 2014

[2] B. Clouet, et al., "Networking Aspects for Next-Generation Elastic Optical Interfaces," JOCN, 2016

[3] J. M. Fabrega, et al., "On the filter narrowing issues in elastic optical networks," JOCN, 2016

[4] T. Rahman, et al., "On the Mitigation of Optical Filtering Penalties Originating from ROADM Cascade," PTL, 2014

[5] C. Delezoide, et al., "Weighted Filter Penalty Prediction for QoT Estimation," OFC, 2018

[6] M.P. Belanger, et al., "Margin requirement of disagg. the DWDM transport sys. and its consequence on application economics," OFC, 2018

[7] https://www.finisar.com/roadms-wavelength-management/focm01fxc1mn

[8] C. Delezoide, et al., "Automated Alignment Between Channel and Filter Cascade," OFC, 2019

[9] K. Christodoulopoulos, et al., "Toward efficient, reliable, and autonomous optical networks: the ORCHESTRA solution [Invited]," JOCN, 2019

[10] A. Mahajan, et al., "Machine Learning Assisted EDFA Gain Ripple Modelling for Accurate QoT Estimation,” ECOC, 2019 